\title{
User Acceptance of QR Code based Mobile Application in Interactive E- Commerce
}

\author{
Yuhanis Yusof*, Lee Cheng Hooi and Azizi Abas \\ School of Computing, Universiti Utara Malaysia, Malaysia \\ yuhanis@uum.edu.my; azizia@uum.edu.my; donlee237768@gmail.com \\ *Correspondence: yuhanis@uum.edu.my
}

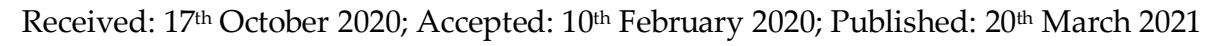

Abstract: Advertisement on products or services can be found in many forms; video, banners, pamphlets, etc. However, these advertisements only function as a one-way communication as consumers are only presented with the product information and the seller. Further action on purchasing the product is absent or is to be made separately. Such an approach may not guarantee a sale for the seller as the customer may only retrieve the information of the product but purchase it elsewhere. Hence, this study demonstrates the use of QR code technology not only in marketing but also in e-commerce. The technology is realized in "On the GoShop" mobile application that has two types of users: seller and buyer. Sellers are people who wanted to promote their product and/or services while buyers are the ones who have a desire to purchase. An evaluation study on the acceptance of the mobile application was then performed on a total of 50 respondents and it is learned that the proposed ecommerce application is well accepted. Ninety percent of the respondents agree with the usefulness of the application and are looking forward to using the application in daily routines. The use of QR code technology not only facilitates consumer in purchasing their desired product but also contribute to cost-effective marketing.

Keywords: QR code; digital marketing; e-commerce; digital advertising

\section{Introduction}

A modern business environment challenges enterprise to be different from their competitors to gain attention from potential customers. Various competition arises in business and these competitors utilize numerous techniques to market their product and/or services. In today's fast-moving era, the size of a business does not matter as similar economic challenges are faced in attracting and retaining customers $[1]$.

It is learned that most product promotions were made using hard copy documents that include flyers, brochures, and pamphlets that are manually distributed to residentials and organizations. Such an approach is not cost-effective as it adds a financial burden to the business operators as well as to the environment. Furthermore, the promotions are a one-way communication as buyers are only provided with the product information. Any action further action on purchasing the desired product need to be made separately by visiting the business outlet. Hence, creating financial and time cost on buyers.

Since quick response code (QR code) has been widely used as product identification, various work has been found to extend it as product descriptor. In line with such movement, this study extends the literature to employ QR code as an interactive e-commerce application. This study demonstrates the use

Yuhanis Yusof, Lee Cheng Hooi and Azizi Abas, "User Acceptance of QR Code based Mobile Application in Interactive ECommerce", Annals of Emerging Technologies in Computing (AETiC), Print ISSN: 2516-0281, Online ISSN: 2516-029X, pp. 102-107, Vol. 5, No. 5, 20 th March 2021, Published by International Association of Educators and Researchers (IAER), DOI: 10.33166/AETiC.2021.05.012, Available: http://aetic.theiaer.org/archive/v5/v5n5/p12.html. 
of QR code technology in e-commerce where sellers can promote their product while buyers can perform the purchase. Information on the product or services is stored in a QR code and the code is used as an advertisement. The QR code image can be printed and distributed either by hardcopy or online. Interested buyers can then scan the code to retrieve the product information. Upon agreement, buyers are then directed to the purchasing process. This approach is realized as an e-commerce application known as "On The Go Shop" and is hoped to facilitate both parties (i.e seller and buyer) as it is costeffective.

\section{QR Code}

Barcode is a graphical image representation, which is capable of storing digital information about an object such as ticketing information, tracking location, unified resource locator, contact list, and many others. Barcode becomes a popular data storage medium as it provides good retrieval accuracy, minimal retrieval time, and cost-effective [2]. There are two categories of the barcode; one- dimensional (1D) and two-dimensional (2D) or also known as matrix barcode [3]. Technically, the difference between these two types of barcodes includes the width of the bar, character set, encoding method, checksum, and specifications [4]. According to a study [5], the 1D barcode is mostly known as "product identification", while the 2D barcode emphasizes "product descriptions". This is because the storage in 1D is limited as compared to the 2D barcode. The 1D barcode contains various widths and parallel space lines while the 2D barcode is represented in the form of a graphical image which is capable to store data horizontally and vertically [6]. Figure 1 shows samples of 1D and 2D barcodes.

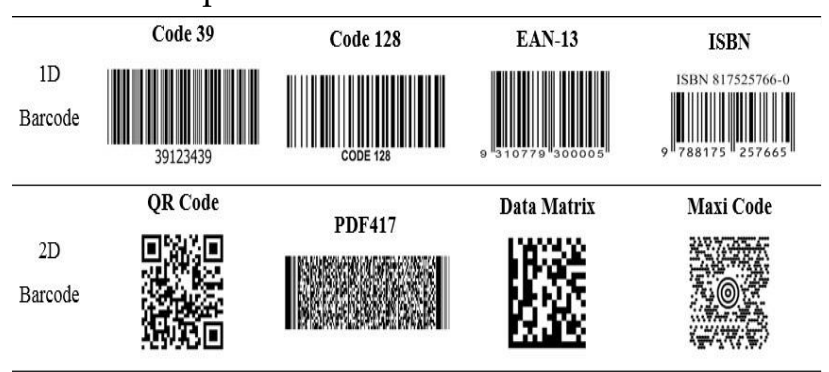

Figure 1. Examples of one-dimensional barcode and two- dimensional barcode [7]

The 2D barcode has two main categories, namely stacked and matrix symbologist. The stacked symbology is developed with two or more small linear rows of barcodes, which are stacked on top of each other. Examples of stacked symbologists are Portable Data File with 4 bars and spaces and each pattern is 17 units long (PDF417), Code 16K, Code 49, and GS1 DataBar [8]. The matrix symbology, on the other hand, is mainly arranged in a grid with the geometric shapes of dark and light colors. It is commonly used in small-item marking, unattended, and high-speed reading applications. Examples of matrix symbologies are Data Matrix, MaxiCode, Aztec Code, Code One, and QR Code [9].

This study focuses on the quick response code (QR code) [11-14] which is categorized under matrix symbology barcode. QR code was first introduced by a Japanese company, Denso Wave Incorporated, in 1994. It was then approved in 2000 as an AIM standard, JIS standard, and ISO standard [7], [14], [15]. Figure 2 shows an example of a QR code.

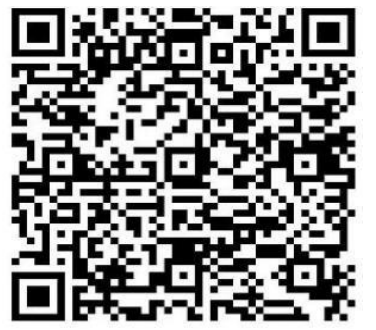

Figure 2. An image of $Q R$ code 
To date, the QR code has the largest capacity storage among 2D barcodes, which is approximately 3 kilobytes [16] and for the coloured barcodes, it has approximately 3 to 100 kilobytes [17]. For all QR code versions, the size of the storage relies on the size of the metric column [18]. The more metric columns exist in a QR code, the more data can be stored in it.

Nowadays, the QR code has been adopted in the areas where 1D barcode was utilized. These applications include retailing, healthcare, life sciences, transportation, office automation, marketing, and advertising [19]. The QR code can transmit information through a print-scan channel [19]-[21] and display the information in the forms of numeric and alphabetic characters, kanji, kana, hiragana, symbols, binary, and control [22]. QR code is very similar to matrix barcode [23], [24], however, the data stored in a QR code is much higher. This leads to a more utilized technology as various data can be stored in it.

Currently, in practice, people use a phone camera to scan the image of a QR code that contains contact information, short messages, authorization to a wireless network, or to open a web page in the telephone's browser that is linked to the webserver [25]. To fully utilize this practice, this study proposes the same action to facilitate the process of purchasing products and/or services. The scanned QR code will lead users to obtain more product information and connects users to the purchasing process.

\section{Methods}

To undertake the study, 5 phases of activities have been completed and this includes theoretical study, data acquisition, application design, and development and evaluation. Figure 3 illustrates the stated phases along with their outcome.

In the first phase, a literature search has been performed to identify problem arises in marketing product and/or services by business operators. It is learned that existing practice is costly and not environmental. Hence, the study proposes the employment of QR code technology in an e-commerce application that facilitates both involved users: seller and buyer. Before designing the application, relevant data on the chosen product were obtained. This has been identified from flyers and pamphlets distributed by the business operator. During the third phase, the collected information is then stored in QR code. In detail, this study realizes QR code in an application known as On the GoShop that is developed to facilitate two groups of users: seller and buyer. As for the seller, the application design includes a marketing medium that allows them to store information about the product or services. Among the functions that can be operated by the seller is the add product, edit product, remove a product, and generate QR Code. The application generates a QR code based on the information provided in the product inventory database.

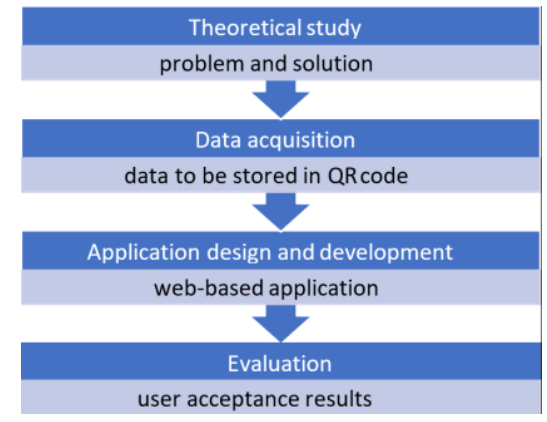

Figure 3. Research phases and outcomes

In the first phase, a literature search has been performed to identify problem arises in marketing product and/or services by business operators. It is learned that existing practice is costly and not environmental. Hence, the study proposes the employment of QR code technology in an e-commerce application that facilitates both involved users: seller and buyer. Before designing the application, relevant data on the chosen product were obtained. This has been identified from flyers and pamphlets distributed by the business operator. During the third phase, the collected information is then stored in 
QR code. In detail, this study realizes QR code in an application known as On the GoShop that is developed to facilitate two groups of users: seller and buyer. As for the seller, the application design includes a marketing medium that allows them to store information about the product or services. Among the functions that can be operated by the seller is the add product, edit product, remove a product, and generate QR Code. The application generates a QR code based on the information provided in the product inventory database.

On the other hand, buyers are consumers who are looking or searching for products or services. A buyer who is interested in a product needs to scan the advertised QR code (i.e through their smart gadget) to retrieve information about the product. To ease the buyer, On the GoShop application will then lead the buyer to a purchasing site that allows him to proceed with the purchase order and payment. In general, a buyer can scan the QR code, submit an order of the desired product, and make the required payment. Once the purchase process has been completed, the product inventory database will be updated to depict the current product availability. Once the On the GoShop application is ready, it was evaluated by 50 users that include 20 sellers and 30 buyers. The main objective of the evaluation was to investigate the usability of On the GoShop from the seller and buyer perspectives. Furthermore, it is also to evaluate the functionality of On the GoShop application.

\section{Results}

In this study, the results of user evaluation focus on ease of use, usefulness, and user satisfaction. Table 1 depicts the ease of use as evaluated by seller and buyer.

Table 1. On the GoShop Application: Ease of Use

\begin{tabular}{l|c|c}
\hline & Seller & Buyer \\
\hline Strongly agree & 10 & 18 \\
\hline Agree & 9 & 12 \\
\hline Neutral & 1 & 0 \\
\hline Disagree & 0 & 0 \\
\hline Strongly disagree & 0 & 0 \\
\hline
\end{tabular}

It is noted that both users evaluated the application as easy to be used. Such an outcome also leads to positive feedback on satisfaction where Table 2 includes the relevant data.

\begin{tabular}{l|c|c}
\multicolumn{2}{c}{ Table 2. On the GoShop Application: User Satisfaction } \\
\hline Strongly agree & Seller & Buyer \\
\hline Agree & 11 & 17 \\
\hline Neutral & 8 & 11 \\
\hline Disagree & 1 & 2 \\
\hline Strongly disagree & 0 & 0 \\
\hline
\end{tabular}

Besides, both types of users also have a similar opinion on the usefulness of On the GoShop. This can be seen in the data depicted in Table 3 where $90 \%$ of both types of respondents agree that the application is useful. Such a positive outcome is due to the capability of On the GoShop that save users time and meets their need. Data in Table 4 depicts the number of users who agree and disagree on the matter.

Table 3. The usefulness of On the GoShop Application

\begin{tabular}{l|c|c}
\hline & Seller & Buyer \\
\hline Strongly agree & 10 & 12 \\
\hline Agree & 8 & 15 \\
\hline Neutral & 2 & 3 \\
\hline Disagree & 0 & 0 \\
\hline Strongly disagree & 0 & 0 \\
\hline
\end{tabular}


Table 4. Benefits of On the GoShop Application

\begin{tabular}{l|l|l|l|l}
\hline & \multicolumn{2}{l}{ Save users time } & \multicolumn{2}{l}{ Meet users' needs } \\
\cline { 2 - 5 } & Seller & Buyer & Seller & Buyer \\
\hline Strongly agree & 14 & 21 & 10 & 16 \\
\hline Agree & 4 & 7 & 9 & 11 \\
\hline Neutral & 2 & 1 & 1 & 3 \\
\hline Disagree & 0 & 1 & 0 & 0 \\
\hline Strongly disagree & 0 & 0 & 0 & 0 \\
\hline
\end{tabular}

\section{Conclusion}

Nowadays, the use of smart gadgets such as handphones can be seen everywhere and at any time. People spend a lot of their time on the internet, either for business or pleasure. Hence, employing QR code in an e-commerce application facilitates users in buying their desired product, especially the ones who are busy and always on the road. This paper provides another means of using QR code in ecommerce. The developed On the GoShop application has received encouraging feedback from the users as QR code is cost-effective for sellers due to the cheap medium of providing consumers with product information. Furthermore, consumers can easily and directly purchase any of the advertised products or services from the seller.

The work presented in this study can further be extended into exciting directions that includes expanding the QR code data storage by using color combination so that more information and various type of information can be stored in it. This may include a video on how the benefits of the product as well as on how to use it. Besides, security issues of using QR code in e-commerce should also be considered.

\section{Acknowledgment}

This work presented in this study has been supported by Universiti Utara Malaysia research grant scheme (S/O code 13976).

\section{References}

[1] Mizani Neda, Sheikh, Reza, Gholami Atena and Sana Shib Shankar, "Attracting and Retaining Customers by Axiomatic Design and Incomplete Rough-Set Theory", International Journal of Applied and Computational Mathematics, 2018, Vol. 4, No. 2, pp. 1-17, DOI: 10.1007/s40819-018-0507-9.

[2] Krassie Petrova, Adriana Romanello, B. Dawn Medlin and Sandra A. Vannoy, "QR Codes Advantages and Dangers", Proceedings of the 13th International Joint Conference on e-Business and Telecommunications, Lisbon, Portugal, 26-28 July, 2016, pp. 112-115, 2016, DOI: 10.5220/0005993101120115.

[3] Feng Xiaofei and Zheng Herong, "Design and realization of 2D color barcode with high compression ratio", Proceedings of International Conference on Computer Design and Applications, Qinhuangdao, China, 25-27 June, 2010, pp. V1-314-V1-317, DOI: 10.1109/ICCDA.2010.5540872.

[4] Elena Purcaru and Christian Tom, "2D Barcode for DNA Encoding", Journal of Mobile Embedded and Distributed Systems, 2011, Vol. 3, No. 3, pp. 142-153.

[5] Chuang Jun Chou, Hu Yu-Chen and Ko Hsien-Ju, "A Novel Secret Sharing Technique Using QR Code", International Journal of Image Processing, 2010, Vol. 4 No. 5, pp. 468-475, Available: https://www.cscjournals.org/manuscript/Journals/IJIP/Volume4/Issue5/IJIP-263.pdf

[6] Lin Jeng-An and Fuh Chiou-Shann, "2D Barcode Image Decoding", Mathematical Problems in Engineering, 2013, Vol. 2013, pp. 1 - 10, DOI: 10.1155/2013/848276.

[7] Rinkalkumar M. Rathod, Kalpesh R. Jadav and Archit Somani, "A Review on 1D \& 2D Barcode with QR Code Basic Structure and Characteristics", International Journal of Futuristic Trends in Engineering and Technology, 2014, Vol. 1, No. 11, pp. 2418-2421, Available: http://www.ijsrd.com/articles/IJSRDV1I11026.pdf.

[8] Zhang Weijun and Yang Tianrui, "An Improved Algorithm for QR Code Image Binarization", Proceedings of 2014 International Conference on Virtual Reality and Visualization, August, IEEE Computer Society, Washington, United States, 2014, pp. 154-159, DOI: 10.1109/ICVRV.2014.51. 
[9] Sutheebanjard, P. and Premchaiswadi W., "QR-code generator", Proceedings of the 8th International Conference on ICT and Knowledge Engineering, Bangkok, Thailand, 24-25 November, IEEE, Piscataway, N.J., 2010, pp. 89-92, DOI: 10.1109/ICTKE.2010.5692920.

[10] Jae Hwa Chang, "An Introduction to Using QR Codes in Scholarly Journals", Science Editing, 2014, Vol. 1, No. 2, pp. 113-117, DOI: 10.6087/kcse.2014.1.113, Available: https://www.escienceediting.org/upload/se-1-2-113.pdf.

[11] Karia Chiraag Thakrar, Hughes Andrew and Carr Sue, "Uses Of Quick Response Codes", In Healthcare Education: A Scoping Review, BMI Medical Education, 2019, Vol. 19, No.456, pp. 1-14, DOI: https://doi.org/10.1186/s12909019-1876-4

[12] Rani Mary Shanthi and Euphrasia K. Rosemary, "Data Security Through Qr Code Encryption And Steganography", Advanced Computing: An International Journal, 2016, Vol. 7, No. 1/2, pp. 1-7.

[13] Sarkar Sayani, Pu Limeng, Wu Hsiao-Chun, Huang Scott C.-H. and Wu Yiyan, "New multimedia archiving technique using multiple quick-response codes", Proceedings of the IEEE International Symposium on Broadband Multimedia Systems and Broadcasting, Italy, 7-9 June, IEEE, 2017, pp. 1-6, DOI: 10.1109/BMSB.2017.7986236.

[14] Melgar Max E. Vizcarra, Zaghetto Alexandre, Macchiavello Bruno and Nascimento Anderson C.A., "CQR codes: Colored quick-response codes", Proceedings of the IEEE International Conference on Consumer Electronics, Berlin, Germany, 3-5 September, IEEE, 2012, pp. 321-325, DOI: 10.1109/ICCE-Berlin.2012.6336526.

[15] Wang Sibing, Yang Tao, Li Jing, Yao Bowei and Zhang Yanning, "Does a QR code must be black and white?", Proceedings of the International Conference on Orange Technologies, Hong Kong, China, 19-22 December, IEEE, 2015, pp. 161-164, DOI: 10.1109/ICOT.2015.7498513.

[16] Grillo Antonio, Lentini Alessandro, Querini Marco and Italiano Giuseppe F., "High capacity colored two dimensional codes", Proceedings of the International Multiconference on Computer Science and Information Technology, Wisla, Poland, 18-20 October, IEEE, 2010, pp. 709-716, DOI: 10.1109/imcsit.2010.5679869.

[17] Hiren J. Galiyawala and Kinjal H. Pandya, "To increase data capacity of QR code using multiplexing with color coding: An example of embedding speech signal in QR code", Proceedings of the 11th IEEE India Conference: Emerging Trends and Innovation in Technology, 11-14 December, Pune, 2015, pp. 1-6, DOI: 10.1109/INDICON.2014.7030441.

[18] Zhang Mu, Yao Dan and Zhou Qian, "The Application and Design of QR Code in Scenic Spot's eTicketing System - A Case Study of Shenzhen Happy Valley", International Journal of Science and Technology, 2012, Vol. 2 No. 12, pp 817-822.

[19] Kinjal H. Pandya and Hiren J. Galijawala, "A Survey on QR Codes in Context of Research and Application", International Journal of Emerging Technology and Advanced Engineering, 2014, Vol. 4 No. 3, pp. 258-262.

[20] Nikolaos T. and Kiyoshi T., "QR-Code Calibration For Mobile Augmented Reality Applications: Linking A Unique Physical Location To The Digital World", ACM SIGGRAPH 2010, Los Angeles, USA, Article 10, DOI: 10.1145/1836845.1836999.

[21] Bhakti Magadum, "Data security in QR code using Steganography", International Journal of Innovative Research in Computer and Communication Engineering, 2017, Vol. 5, No. 5, pp. 10058-10063, DOI: 10.15680/IJIRCCE.2017. 0505308

[22] Kan Tan-Wei, Teng Chin-Hung and Chou Wen-Shou, "Applying QR code in augmented reality applications", Proceedings of 8th International Conference on Virtual Reality Continuum and its Applications in Industry, December, Yokohama, Japan, ACM, 2009, pp. 253-257, DOI: 10.1145/1670252.1670305.

[23] Dita Ion-Cosmin, Otesteanu Marius and Quint Franz, "Data Matrix Code - A reliable optical identification of microelectronic components", Proceedings of IEEE 17th International Symposium for Design and Technology of Electronics Packages, 20-23 October 2011, Timisoara, Romania, pp. 39-44, DOI: 10.1109/SIITME.2011.6102683.

[24] Gao Jerry, Kulkarni Vijay, Ranavat Himanshu, Chang Lee and Mei Hsing, "A 2D barcode-based mobile payment system", Proceedings of the 3rd International Conference on Multimedia and Ubiquitous Engineering, 4-6 June 2009, Qingdao, China, IEEE, pp. 320-329, DOI: 10.1109/MUE.2009.62. DOI: 10.1109/MUE.2009.62.

[25] Gutierrez Francisco, Abud M. Antonieta, Vera Fernando and Sanchez J. Alfredo, "Application of contextual QR codes to augmented reality technologies", Proceedings of the 23rd International Conference on Electronics, Communications and Computing, 11-13 March 2013, Cholula, Mexico, pp. 264-269, DOI: 10.1109/CONIELECOMP.2013.6525798.

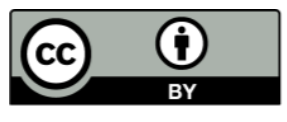

(C) 2021 by the author(s). Published by Annals of Emerging Technologies in Computing (AETiC), under the terms and conditions of the Creative Commons Attribution (CC BY) license which can be accessed at http://creativecommons.org/licenses/by/4.0. 